\title{
Flächengitter in geschmolzenem Zinn und Silber sowie in festem amorphem Selen nach der Fourier-Analyse
}

\author{
H. Richter und G. Breitling
}

\author{
Max-Planck-Institut für Metallforschung, Stuttgart \\ und Medizinisches Strahleninstitut der Universität Tübingen \\ (Z. Naturforschg. 21 a, 1710—1718 [1966] ; eingegangen am 27. Juni 1966)
}

Die $4 \pi r\left[\varrho(r)-\varrho_{0}\right]$-Kurven von geschmolzenem $\mathrm{Sn}$ bei $250^{\circ}$ und $750^{\circ} \mathrm{C}$ zeigen, daß in der Schmelze zwei Strukturen vorkommen, die dichteste Kugelpackung mit $r_{1}=3,15$ bzw. $3,18 \AA$ (Literaturwert $r_{12}=3,16 \AA$ ) und die Flächengitter-Struktur mit $r_{1}^{\prime}=3,01$ bzw. 3,03 $\AA$ als Kantenlänge einer quadratischen Gitterzelle. Dabei ist $r_{1}{ }^{\prime} \approx\left[r_{1}\right]_{G}=3,02 \AA$, also gleich dem kürzesten Gitterabstand. In der Ag-Schmelze tritt die dichteste Kugelpackung in Form von Flächengittern mit der (111) kub.- bzw. (001) hex.-Ebene als Basis auf. Die Abmessungen der zugehörigen hexagonalen Gitterzelle sind $r_{1}=2,94 \AA$ und $\varphi=120^{\circ}$. Uberraschenderweise zeichnen sich in der $4 \pi r\left[\varrho(r)-\varrho_{0}\right]$ Kurve lediglich die Abstände der Zickzackkette ab, die von den Atomabständen $r_{1}=2,94 \AA$ und $r_{1} \sqrt{3}=5,09 \AA$ gebildet wird. Die Flächengitter-Struktur besteht bei Ag wie bei $\mathrm{Sn}$ aus einer quadratischen Gitterzelle, und zwar mit $r_{1}{ }^{\prime}=2,72 \AA$ als Kantenlänge. Danach ist die Ag-Schmelze ein Haufwerk von Flächengittern mit hexagonaler und mit quadratischer Gitterzelle; Gleiches gilt für die Schmelzen von $\mathrm{Au}, \mathrm{Pb}$ und $\mathrm{Tl}$. Auch in den $4 \pi r\left[\varrho(r)-\varrho_{0}\right]$-Kurven von geschmolzenem $\mathrm{Na}$ und Cs bildet sich die Zickzackkette der dichtesten Kugelpackung ab. Die Alkalimetallschmelzen stehen damit dem geschmolzenen $\mathrm{Au}, \mathrm{Ag}, \mathrm{Pb}$ und $\mathrm{Tl}$ sehr nahe, weniger dagegen dem geschmolzenen $\mathrm{Sn}, \mathrm{Ga}$ und $\mathrm{Bi}$.

Die Zickzackkette wird auch bei den festen amorphen Stoffen erhalten. So zeichnet sich in der $4 \pi r^{2}\left[\varrho(r)-\varrho_{0}\right]$-Kurve von festem amorphem Se die ebene Zickzackkette ab, die sich aus den Gitter-

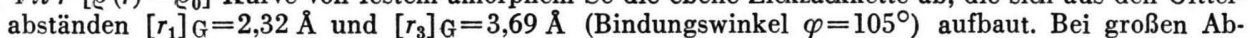
standswerten ist selbst hier wegen der Lagestreuung der Atome eine deutliche Abstandsverkürzung wie bei geschmolzenem $\mathrm{Au}, \mathrm{Ag}, \mathrm{Pb}$ und $\mathrm{Tl}$ zu beobachten.

Zur Bestimmung der Atomverteilung in nichtkristallinen Stoffen nach der FourIER-Analyse der Intensitätskurve geht man von der Gleichung

$4 \pi r^{2} \varrho(r)=4 \pi r^{2} \varrho_{0}+\frac{2 r}{\pi} \int_{0}^{\infty} s i(s) \cdot \sin (s r) \mathrm{d} s$

aus; hierin bezeichnet $4 \pi r^{2} \varrho_{0}$ den Beitrag der gleichmäßigen (ungeordneten) Atomverteilung und das Integral $2 r / \pi \int_{0}^{\infty} s i(s) \cdot \sin (s r) \mathrm{d} s$ den der diskreten (geordneten) Atomverteilung. Nimmt man im einfachsten Falle für $i(s)$ die ungedämpfte Interferenzfunktion

$$
i(s)=\left(I(s)-N f^{2}\right) / N f^{2}=\sin \left(s r_{i}\right) / s r_{i}
$$

an, dann geht $\mathrm{Gl}$. (1) über in

$$
4 \pi r^{2} \varrho(r)=4 \pi r^{2} \varrho_{0}+\frac{2 r}{\pi r_{i}} \int_{0}^{\infty} \sin \left(s r_{i}\right) \cdot \sin (s r) \mathrm{d} s
$$

oder nach Integration und Division durch $r$

$$
4 \pi r\left[\varrho(r)-\varrho_{0}\right]=\frac{1}{\pi r_{i}}\left[s \frac{\sin \left(r_{i}-r\right) s}{\left(r_{i}-r\right) s}-s \frac{\sin \left(r_{i}+r\right) s}{\left(r_{i}+r\right) s}\right]_{s_{1}}^{s_{2}}
$$

mit $s_{1}$ und $s_{2}$ als Integrationsgrenzen ${ }^{1}$. Nach Gl. (2) bestimmt der vorgegebene Atomabstand $r_{i}$ die Periode der Interferenzfunktion $\sin \left(s r_{i}\right) / s r_{i}$, zum anderen muß er auf der rechten Seite der Gl. (3) bzw. in der zugehörigen $4 \pi r\left[\varrho(r)-\varrho_{0}\right]$-Kurve als Atomabstand auftreten. Hier legt er nach Richter, Breituing und Herre ${ }^{2}$ die Abszisse des Hauptmaximums fest. Damit ist allein die $4 \pi r\left[\varrho(r)-\varrho_{0}\right]$-Kurve geeignet, die Atomabstände unverfälscht bis zu großen $\mathrm{Ab}$ -

1 Unmittelbar erhält man Gl. (3) aus dem Intensitätsausdruck

$$
I(s)=N f^{2}\left(1+\int_{0}^{\infty} 4 \pi r^{2}\left[\varrho(r)-\varrho_{0}\right][\sin (s r) / s r] \mathrm{d} r\right.
$$

bzw. aus

$$
s \cdot i(s)=\int_{0}^{\infty} \frac{4 \pi r^{2}\left[\varrho(r)-\varrho_{0}\right]}{r} \cdot \sin (s r) \mathrm{d} r .
$$

Nach dem Fourierschen Umkehrsatz ist nämlich

$$
4 \pi r\left[\varrho(r)-\varrho_{0}\right]=\frac{2}{\pi} \int_{0}^{\infty} s i(s) \cdot \sin (s r) \mathrm{d} s .
$$

Die Abweichungen der Atomabstände nach der $4 \pi \mathrm{r}[\varrho(r)$ $\left.-\varrho_{0}\right]$ - und nach der $4 \pi r^{2}\left[\varrho(r)-\varrho_{0}\right]$-Darstellung sind im allgemeinen kleiner als $1 \%$. Bei der $4 \pi r^{2}\left[\varrho(r)-\varrho_{0}\right]$-Darstellung treten allerdings die Maxima bei großen $r$-Werten deutlicher hervor.

2 H. Richter, G. Breithing u. F. Herre, Z. Angew. Phys. 8, 433 [1956]. - F. Herre u. H. Richter, Z. Phys. 150, 149 [1958]. 
standswerten zu liefern (vgl. BreItLing und RichteR ${ }^{3}$ ). Die $4 \pi r^{2} \varrho(r)$-Kurve ergibt dagegen die Atomzahlen der geordneten und der ungeordneten Bereiche, während die $4 \pi r^{2}\left[\varrho(r)-\varrho_{0}\right]$-Kurve die Atomzahlen der geordneten Bereiche mit ihrer diskreten Atomverteilung liefert.

Aus früheren Untersuchungen liegen bereits $4 \pi r\left[\varrho(r)-\varrho_{0}\right]$-Kurven von geschmolzenem Sn bei $250^{\circ}$ und $750^{\circ} \mathrm{C}$ sowie von geschmolzenem $\mathrm{Ag}$ vor, sie sollen kurz besprochen werden. Wieder sind die Maxima mit 1., 2., 3. usf. Maximum bezeichnet, wenn sie aber durch Überlagerung zustande kommen, sind sie mit I., II., II. usf. Maximum gekennzeichnet. Wie früher wird auch hier die dichteste Kugelpackung häufig als Kugelmodell-Struktur bezeichnet, dabei ist eine räumliche Anordnung der Atome angenommen.

\section{I a. Geschmolzenes Zinn bei $250^{\circ} \mathrm{C}$}

In Abb. $1 \mathrm{a}$ ist die $4 \pi r\left[\varrho(r)-\varrho_{0}\right]$-Kurve einer Sn-Schmelze von $250{ }^{\circ} \mathrm{C}$ dargestellt; sie zeigt vier ausgeprägte Maxima. Das I. Maximum tritt bei

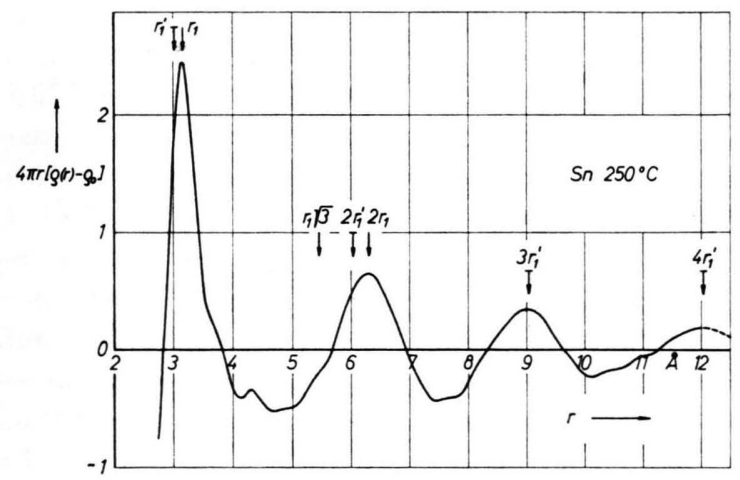

Abb. 1 a. $4 \pi r\left[\varrho(r)-\varrho_{0}\right]$-Kurve von geschmolzenem Zinn bei $250^{\circ} \mathrm{C}$; Integrationslänge $s_{2}=11,5$. Berechnete Pfeillagen $\downarrow$ bei $r=r_{1}^{\prime}, 2 r_{1}^{\prime}$ und $4 r_{1}{ }^{\prime}$ mit $3 r_{1}{ }^{\prime}=9,03 \AA$ oder mit $r_{1}^{\prime}=3,01 \AA$ sowie $\downarrow$ bei $r=r_{1} \sqrt{3}$ und $2 r_{1}$ mit $r_{1}=3,15 \AA$.

$r_{\mathrm{I}} \approx r_{1}=3,15 \AA(\downarrow)$, dem kürzesten Atomabstande der dichtesten Kugelpackung (Literaturwert: $r_{12}=$ $3,16 \AA$ ), auf und das II. Maximum bei $r_{\mathrm{II}}=6,29 \AA$, dem doppelten Atomabstande $2 r_{1}=2 \cdot 3,15 \AA=$ $6,30 \AA$ der Kugelmodell-Struktur. Der Abszissenwert des 3. Maximums bei $r=9,03 \AA=3 r_{1}{ }^{\prime}$ ergibt als kürzesten Atomabstand der FlächengitterStruktur $r_{1}{ }^{\prime}=3,01 \AA(\downarrow)$. Dieser Atomabstand stimmt

3 G. Breitling u. H. Richter, Z. Naturforschg. 1967.

4 H. Richter u. D. Handtmann, Z. Phys. 181, 206 [1964]. gut mit dem kürzesten Gitterabstand $\left[r_{1}\right]_{\mathrm{G}}=3,02 \AA$ überein. Die $4 \pi r\left[\varrho(r)-\varrho_{0}\right]$-Kurve in Abb. 1 a liegt nur bis $12 \AA$ vor. Die letzten drei Meßpunkte bei $r=11,90 ; 11,95$ und $12,00 \AA$ deuten an, da $\beta$ das 4. Maximum bei $r=12,04 \AA=4 \cdot 3,01 \AA$ auftritt. Der Atomabstand $r_{1}{ }^{\prime}=3,01 \AA$ wird also auch durch dieses Maximum nahegelegt. Nach Abb. 1 a wird der Verlauf der $4 \pi r\left[\varrho(r)-\varrho_{0}\right]$-Kurve im hinteren Teile vom dreifachen und vom vierfachen Atomabstande $3 r_{1}{ }^{\prime}$ und $4 r_{1}{ }^{\prime}$ der Flächengitter-Struktur bestimmt, im vorderen Kurventeile (I. und II. Maximum) dagegen praktisch allein vom einfachen und vom doppelten Atomabstande $r_{1}$ und $2 r_{1}$ der Kugelmodell-Struktur. Die Atomabstände $r_{1}{ }^{\prime}$ und $2 r_{1}{ }^{\prime}$ der FlächengitterStruktur haben mitunter eine geringfügige Verschiebung der Maxima, insbesondere des II. Maximums, zur Folge. Man hat dabei zu beachten, daß Vielfache des kürzesten Atomabstandes $\left[r_{1}\right]_{\mathrm{G}}=3,02 \AA \mathrm{im} \mathrm{Sn}$ Gitter nicht auftreten. Danach sind die Flächengitter mit $r_{1}{ }^{\prime}=3,01 \AA$ als kürzestem Abstand und mit Debye-Streuung, wie sie in der Sn-Schmelze vorkommen, keine Restbestände des Gitters, sie haben mit dem Sn-Gitter lediglich den kürzesten Atomabstand gemeinsam.

Die kürzesten Atomabstände $r_{1}=3,15 \AA$ und $r_{1}^{\prime}=3,01 \AA$ der Kugelmodell- und der FlächengitterStruktur, wie sie die Fourier-Analyse der Intensitätskurve liefert, stimmen bestens mit den Ergebnissen der sukzessiven Analyse von Richter und HandTMANN ${ }^{4}$ überein, welche die Abstandswerte $r_{1}=3,15 \AA$ und $r_{1}{ }^{\prime}=3,02 \AA$ ergibt. Damit zeigt auch die FourierAnalyse, daß in der Sn-Schmelze neben der dichtesten Kugelpackung eine zweite Struktur mit dem kürzesten Atomabstand $r_{1}{ }^{\prime}=3,01 \AA$ vorliegt, sie streut wie die Kugelmodell-Struktur nach Debye ${ }^{5}$.

Bezüglich der Struktur der Sn-Schmelze läßt sich folgendes sagen: In der Sn-Schmelze kommen kleinste Bereiche mit dichtester Kugelpackung $\left(r_{1}=3,15 \AA\right)$ vor, daneben sind ausgedehnte Teilbereiche des SnGitters in Form von Flächengittern [ (001) tetr.-Ebene $=$ Basisebene] mit Streuung nach v. LauE ${ }^{6}$ vorhanden und zudem noch einfach-primitive kubische, höchstwahrscheinlich aber einfach-primitive quadratische Gitterzellen (Flächengitter) mit $r_{1}{ }^{\prime}=\left[r_{1}\right]_{\text {Flg. }}$. $=\left[r_{1}\right]_{\text {Rg. }}=3,01 \AA$ als kürzestem Atomabstand wie im Sn-Gitter. Die quadratischen Gitterzellen sind damit gitterverwandt; sie sind in ihrem Aufbau gestört,

5 P. Debye, Ann. Phys. 46, 809 [1915].

6 M. v. LAUE, Z. Kristallogr. 82, 127 [1932]. 
und streuen wie die Bereiche der Kugelmodell-Struktur nach Debye. Die Struktur dieser gestörten Gitterzellen wird sinngemäß als „Flächengitter-Struktur“ bezeichnet. Solche gitterverwandte Zellen sind auch in festem amorphem und in geschmolzenem $\mathrm{Bi}^{7}$ beobachtet worden. Die Flächengitter in den Schmelzen von $\mathrm{Au}, \mathrm{Ag}, \mathrm{Pb}$ und $\mathrm{Tl}$ besitzen den gleichen Aufbau, nur ist hier $r_{1}{ }^{\prime}<\left[r_{1}\right]_{\mathrm{G}}=r_{12}$, sie streuen lediglich nach Debye. Die Struktur dieser FlächengitterBereiche ist gitterfremd.

Die einfach-primitive kubische Gitterzelle von festem amorphem und von geschmolzenem $\mathrm{Bi}$, und zwar zum Flächengitter abgebaut, stellt den Prototyp der Flächengitter-Struktur der Metallschmelze dar. Bei Bi sind die Flächengitter ausgedehnt und geordnet, sie streuen daher nach v. LAUE, bei Störung dagegen nach Deвye. Im letzten Falle heben sich allein die Atomabstände $r_{v}{ }^{\prime}=v \cdot r_{1}{ }^{\prime}$ mit $r_{1}{ }^{\prime}=\left[r_{1}\right]_{\mathrm{G}}$ $=3,10 \AA$ in der Atomverteilungskurve ab. Im Gegensatz dazu sind im Bi-Gitter Vielfache des kürzesten Gitterabstandes $\left[r_{1}\right]_{G}$ nicht möglich. Die Flächengitter-Struktur bei geschmolzenem $\mathrm{Au}, \mathrm{Ag}, \mathrm{Pb}, \mathrm{Tl}$ u.a.m. (Gruppe I) sowie bei $\mathrm{Hg}, \mathrm{Sn}, \mathrm{Ga}, \mathrm{Ge}$ u. a.m. (Gruppe II) baut sich in gleicher Weise auf, doch streuen die Flächengitter der Gruppe I ausschließlich nach Debye. Wie bei den Flächengittern von $\mathrm{Bi}$ ist auch hier $r_{v}{ }^{\prime}=v \cdot r_{1}{ }^{\prime}$; dabei ist bei der Gruppe I der kürzeste Atomabstand $r_{1}{ }^{\prime}<\left[r_{1}\right]_{\mathrm{G}}=r_{12}$, bei der Gruppe II dagegen $r_{1}^{\prime} \approx\left[r_{1}\right]_{\mathrm{G}}$.

Von den Atomabständen der Flächengitter-Struktur treten lediglich die Abstände $r_{v}{ }^{\prime}=v \cdot r_{1}{ }^{\prime}$ mit $v=1$, 2, 3 usf. in der Atomverteilungskurve auf (vgl. die Abb. 1 a und b). Bezüglich der Abstände $r_{v}{ }^{\prime}$ sind die Atome lückenlos auf einer Geraden angeordnet; für diese Atome sind die Abstandsschwankungen, verglichen mit denen der seitlich dazu gelegenen Atome (Seitenatome), am geringsten. Diese Atome zeichnen sich daher in der Atomverteilungskurve bevorzugt ab. Von den Atomabständen $r_{v}^{\prime}$ kommt in den Gittern von Sn und Bi nur der kürzeste Abstand $r_{1}^{\prime} \approx\left[r_{1}\right]_{\mathrm{G}}$ vor.

7 Aus dem Auftreten der Atomabstände $r_{v}{ }^{\prime}=v \cdot r_{1}{ }^{\prime}$ in den $4 \pi r\left[\varrho(r)-\varrho_{0}\right]$-Kurven der Abb. $1 \mathrm{a}$ und b könnte man auf das Vorliegen von Atomketten in der Sn-Schmelze schließen. Bei festem amorphem und bei geschmolzenem Bi sind aber die Bereiche mit gitterverwandter Struktur teilweise so stark ausgedehnt und geordnet, daß sie nach v. Laue streuen. Dieser Befund spricht gegen das Vorhandensein von Atomketten mit $r_{1}{ }^{\prime}=\left[r_{1}\right]_{G}$ als kürzestem Atomabstand. Fujime $^{8}$ gibt die Interferenzlagen für das feste

\section{I b. Geschmolzenes Zinn bei $750{ }^{\circ} \mathrm{C}$}

Die $4 \pi r\left[\varrho(r)-\varrho_{0}\right]$-Kurve einer Sn-Schmelze von $750{ }^{\circ} \mathrm{C}$ in Abb. $1 \mathrm{~b}$ zeigt drei ausgeprägte Maxima. Das I. Maximum bei $r_{\mathrm{I}}=3,10 \AA$ ist in Übereinstimmung mit Richter und Handtmann ${ }^{4}$ [vgl. S. 229,

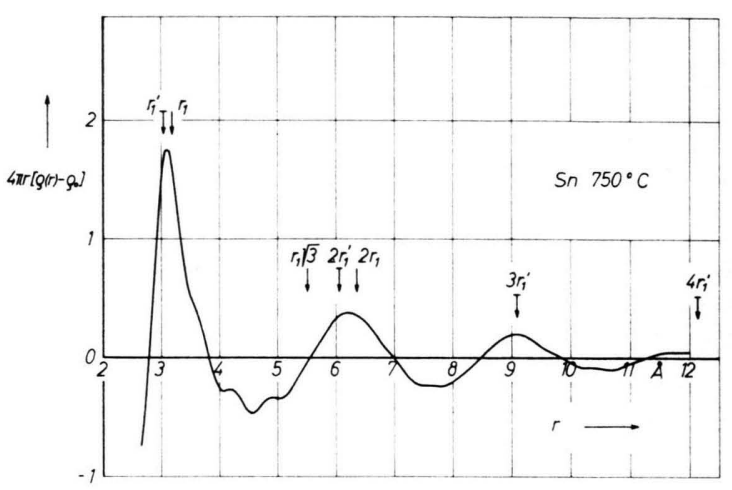

Abb. 1 b. $4 \pi r\left[\varrho(r)-\varrho_{0}\right]$-Kurve von geschmolzenem Zinn bei $750{ }^{\circ} \mathrm{C}$; Integrationslänge $s_{2}=11,3$. Berechnete Pfeillagen $\downarrow$ bei $r=r_{1}{ }^{\prime}, 2 r_{1}^{\prime}$ und $4 r_{1}^{\prime}$ mit $3 r_{1}^{\prime}=9,08 \AA$ oder mit $r_{1}^{\prime}=3,03 \AA$ sowie $\downarrow$ bei $r=r_{1} \sqrt{3}$ und $2 r_{1}$ mit $r_{1}=3,18 \AA$.

Fig. 10 b; Kombination der Gaussschen Wahrscheinlichkeitsfunktion mit den Ergebnissen der sukzessiven Analyse] trotz derselben Integrationslänge $s_{2}$ wie in Abb. 1 a nach kleinen $r$-Werten verschoben, und zwar durch den erheblichen Zuwachs an Flächengitterbereichen mit Debye-Streuung. Richter und HandtMANN ${ }^{4}$ finden bei geschmolzenem $\mathrm{Sn}$ von $750{ }^{\circ} \mathrm{C}$ $r_{1}=3,18 \AA(\downarrow)$ und $r_{1}{ }^{\prime}=3,04 \AA$ als kürzeste Atomabstände der Kugelmodell- und der FlächengitterStruktur. Das II. Maximum tritt bei $r_{\mathrm{II}}=6,22 \AA$ auf, häufig bei $r_{I I} \approx 6,15 \AA$; auch dieses Maximum ist durch den verstärkten Anteil an Flächengitter-Struktur nach kleinen $r$-Werten verlagert. Wie in Abb. 1 a zeichnet sich auch hier der $r_{1} \sqrt{3}$-Abstand $\left(r_{1} \sqrt{3}=\right.$ $3,18 \sqrt{3} \AA=5,51 \AA$ ) der dichtesten Kugelpackung nicht ab. Das 3. Maximum bei $r=9,08 \AA=3 \cdot r_{1}{ }^{\prime}$ liefert als kürzesten Atomabstand der FlächengitterStruktur $r_{1}^{\prime}=3,03 \AA(\downarrow)$, in guter Übereinstimmung mit Richter und Handtmann ${ }^{4}$. Auch hier wird wie bei geschmolzenem $\mathrm{Sn}$ von $250{ }^{\circ} \mathrm{C}$ der Verlauf der

amorphe $\mathrm{Bi}$ an, sie sind allerdings der Intensitätskurve entnommen. Hieraus folgen als Abmessungen der rhombischen (zweidimensionalen) Gitterzelle $a$ Flg. $=r_{1}{ }^{\prime} \approx 3,14 \AA$ sowie $\varphi_{\mathrm{Flg}} \approx 93^{\circ}$ und $89^{\circ}$, in guter Übereinstimmung mit Richter und Breitling ${ }^{9}$, die $a_{\text {Flg. }}=r_{1}{ }^{\prime}=\left[r_{1}\right]_{\mathrm{G}}=3,10 \AA$ und $\varphi$ Flg. $=94^{\circ}$ bzw. $86^{\circ}$ finden.

8 S. Fujime, Japanese J. Appl. Phys. 5, 59 [1966].

9 H. Richter u. G. Breitling, Fortschr. Phys. 14, 71 [1966]. 
$4 \pi r\left[\varrho(r)-\varrho_{0}\right]-$ Kurve im hinteren Teile vom dreifachen und vom vierfachen Atomabstande $3 r_{1}{ }^{\prime}$ und $4 r_{1}{ }^{\prime}$ der Flächengitter-Struktur festgelegt, im vorderen Kurventeile (I. und II. Maximum) sind dagegen die einfachen und die doppelten Atomabstände $r_{1}$ und $r_{1}{ }^{\prime}$ sowie $2 r_{1}$ und $2 r_{1}^{\prime}$ der Kugelmodell- und der Flächengitter-Struktur bestimmend.

Die Fourier-Analyse der Intensitätskurve von geschmolzenem Sn bestätigt somit die Ergebnisse der neuen Auswertungsverfahren wie der $r_{\mathrm{I}}$-Methode, der abschnittsweisen Fourier-Analyse und der sukzessiven Analyse. Damit sprechen auch die Ergebnisse der Fourier-Analyse für das Nebeneinander zweier Strukturen mit Debye-Streuung, der Kugelmodell- und der Flächengitter-Struktur, in der SnSchmelze.

\section{Geschmolzenes Silber bei $\approx 1000{ }^{\circ} \mathrm{C}$}

In Abb. 2 ist die $4 \pi r\left[\varrho(r)-\varrho_{0}\right]$-Kurve von geschmolzenem Ag dargestellt, sie wurde durch Integration der $i(s)_{\text {exp. }}$-Kurve von Pfannenschmid ${ }^{10} \mathrm{er}$ -

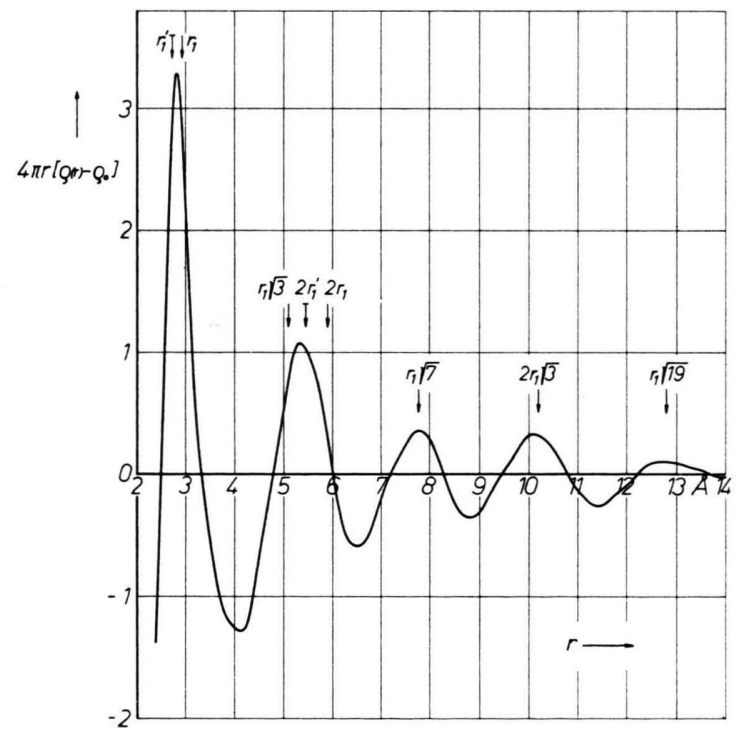

Abb. 2. $4 \pi r\left[\varrho(r)-\varrho_{0}\right]-$ Kurve von geschmolzenem Silber; obere Integrationsgrenze $s_{2}=10,5$. Berechnete Pfeillagen $\downarrow$ bei $r=r_{1} \sqrt{3}, 2 r_{1}, r_{1} \sqrt{7,} 2 r_{1} \sqrt{3}$ und $r_{1} \sqrt{19}$ mit $r_{1}=2,94 \AA$ sowie $\downarrow$ bei $r=2 r_{1}{ }^{\prime}$ mit $r_{1}^{\prime}=2,72 \AA$.

halten. Das I. Maximum tritt bei $r_{\mathrm{I}}=2,82 \AA(2,86 \AA)$ auf, also zwischen dem kürzesten Atomabstande $r_{1}{ }^{\prime}=2,72 \AA(\downarrow)$ der Flächengitter-Struktur und dem

10 O. Pfannenschmid, Dissertation, Techn. Hochschule Stuttgart 1959; Z. Naturforschg. 15 a, 603 [1960]. kürzesten Atomabstande $r_{1}=2,94 \AA(\downarrow)$ der dichtesten Kugelpackung. Die Zahlenwerte in den runden Klammern bezeichnen die der $4 \pi r^{2} \varrho(r)$-Kurve entnommenen Abstandswerte. Beim II. Maximum sind die Verhältnisse ähnlich. Seine Lage bei $r_{\text {II }}=5,33 \AA$ $(5,66 \AA)$ schwankt zwischen den Abstandswerten $r_{1} \sqrt{3}=2,94 \sqrt{3} \AA=5,09 \AA$ und $2 r_{1}{ }^{\prime}=2 \cdot 2,72 \AA=$ $5,44 \AA$; häufig ist das II. Maximum noch stärker als in Abb. 2 in Richtung des doppelten Atomabstandes $2 r_{1}{ }^{\prime}$ der Flächengitter-Struktur verschoben. Der doppelte Atomabstand $2 r_{1}=2 \cdot 2,94 \AA=5,88 \AA$ der Kugelmodell-Struktur ist hier im Gegensatz zum geschmolzenen Sn nicht an der Fixierung des II. Maximums beteiligt. Das 3. Maximum bei $r=7,79 \AA$ ( $\approx 8,30 \AA$ ) läßt sich als $r_{1} \sqrt{7}$-Abstand der kubischdichtesten Kugelpackung $\left(r_{7}=r_{1} \sqrt{7}=2,94 \sqrt{7} \AA=\right.$ $7,78 \AA$ ) mit $N_{7}=48$ Atomen deuten. In der $\mathrm{Ag}$ Schmelze liegen demnach kleinste Bereiche mit Gitterstruktur vor. Die Deutung der übrigen Maxima erfolgt später.

In Tab. 1 sind die Ergebnisse der Fourier-Analyse der zur Abb. 2 gehörigen $i(s)$ exp.-Kurve von geschmolzenem Ag für verschiedene Integrationslängen $s_{2}$ zusammengestellt. Man sieht, daß mit wachsender Integrationslänge $s_{2}$ das I. Maximum bei kleineren $r$-Werten auftritt, daß sich aber die Lagen der übrigen Maxima (Atomabstände) trotz verschiedener Integrationslänge $s_{2}$ nur wenig ändern; sie erfahren höchstens eine geringfügige Abstandsverkürzung, die allerdings um so größer ist, je größer $r$ ist. Die Abstandswerte des $r_{1} \sqrt{7}$-Abstandes streuen am wenigsten und stimmen mit dem berechneten Werte bestens überein. Auch in den $4 \pi r\left[\varrho(r)-\varrho_{0}\right]$-Kurven von geschmolzenem $\mathrm{Au}, \mathrm{Pb}$ und $\mathrm{Tl}$ zeichnet sich der $r_{1} \sqrt{7 \text { - }}$ Abstand sehr deutlich ab und steht ebenfalls mit dem berechneten Werte in bestem Einklang. Beim Übergange zur $4 \pi r^{2} \varrho(r)$-Darstellung werden die Maxima nach großen $r$-Werten verlagert und abgeflacht, und zwar um so mehr, je größer $r$ ist; dabei wird bereits der $r_{1} \sqrt{7}$-Abstand ausgelöscht. Man sieht, daß auch die $4 \pi r\left[\varrho(r)-\varrho_{0}\right]$-Kurve in Abb. 2 für das Vorhandensein zweier Strukturen, der KugelmodellStruktur und der Flächengitter-Struktur, in kleinsten Bereichen der Ag-Schmelze spricht.

Nach Abb. 2 liegen in geschmolzenem Ag kleinste Gitterbereiche vor. Hierbei erhebt sich die Frage: Warum zeichnet sich beim Vorliegen von Gitterbereichen in der Ag-Schmelze der Atomabstand $\left[r_{5}\right]_{\mathrm{G}}=r_{1} \sqrt{5}=2,94 \sqrt{5} \AA=6,57 \AA$ mit 24 Atomen in der $4 \pi r\left[g(r)-\varrho_{0}\right]$-Kurve nicht ab? Selbst, wenn 


\begin{tabular}{|c|c|c|c|c|}
\hline \multicolumn{3}{|c}{ Experimentelle Atomabstände } & \multicolumn{2}{c|}{ Berechnete Atomabstände } \\
\cline { 1 - 2 } \cline { 5 - 5 } 1,6 bis 9,1 & 1,6 bis 10,5 & 1,6 bis 12,7 & Kug.-Struktur & $\begin{array}{c}\text { Flächeng.- } \\
\text { Struktur }\end{array}$ \\
\hline 2,85 & 2,82 & 2,79 & $r_{1}=2,94$ & $r_{1}^{\prime}=2,72$ \\
5,43 & 5,33 & 5,33 & $r_{1} \sqrt{3}=5,09$ & $2 r_{1}^{\prime}=5,44$ \\
\hline 7,80 & 7,79 & 7,81 & $r_{1} \sqrt{7}=7,78$ & \\
10,14 & 10,11 & 10,22 & $2 r_{1} \sqrt{3}=10,18$ & \\
12,67 & 12,75 & 12,73 & $r_{1} \sqrt{19}=12,81$ & \\
\hline
\end{tabular}

Tab. 1. Experimentelle und berechnete Atomabstände in geschmolzenem Silber bei Fourier-Analyse der $i(s)$ exp.-Kurve mit $s_{1}=1,6$ und $s_{2}=9,1 ; 10,5$ bzw. 12,7 als Integrationsgrenzen.

man eine hexagonal-dichteste Packung der Atome annimmt, ist das Problem das gleiche. Hier ist nämlich $r_{7}=r_{1} \sqrt{5}=6,57 \AA$ und $N_{7}=12$ bzw. $r_{8}=r_{1} \sqrt{5^{2} / 3}$ $=7,00 \AA$ und $N_{8}=12$. Als Lösung bleibt die Annahme einer zweidimensional-periodischen Anordnung der Atome bei dichtester Packung, also einer Atomanordnung wie in der (111) kub.-Ebene des AgGitters bzw. wie in der mit ihr identischen $(001)_{\text {hex.- }}$ Ebene des hexagonalen Gitters. In einem solchen Flächengitter treten auf: Der kürzeste Atomabstand $r_{1}$, der $r_{1} \sqrt{3}$-Abstand und deren Vielfache, und zwar jeweils mit 6 nächsten Nachbarn, sowie die weiteren Atomabstände $r_{1} \sqrt{7}, r_{1} \sqrt{13}, r_{1} \sqrt{19}, r_{1} \sqrt{21}, r_{1} \sqrt{31}$, $r_{1} \sqrt{37} \ldots$ und Vielfache hiervon mit je 12 nächsten

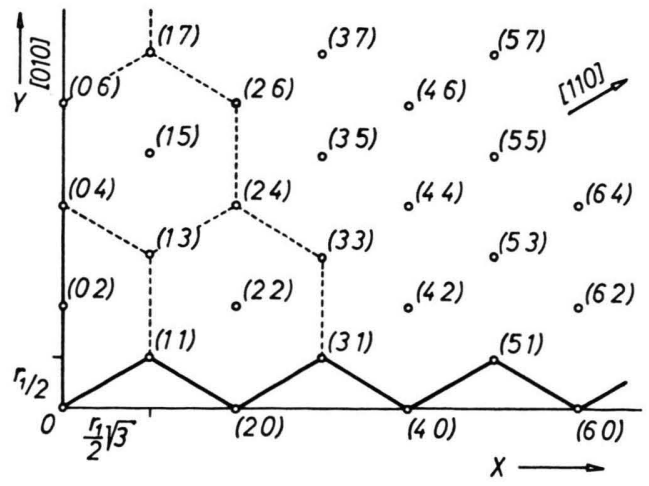

Abb. 3. Indizesschema für die Atomlagen in der (111) kub.bzw. $(001)_{\text {hex. }}$-Ebene bei dichtester Kugelpackung.
Nachbarn. Der $r_{1} \sqrt{5}$-Abstand kommt in diesem Flächengitter nicht vor.

Um die vorliegenden Verhältnisse leichter überblicken zu können, sind in Abb. 3 die Atomlagen der (111) kub.- bzw. (001) hex.-Ebene durch ein zweigliedriges Indizesschema dargestellt. Als Längeneinheit wurde auf der $x$-Achse $\frac{1}{2} \cdot r_{1} \sqrt{3}$ und auf der $y$-Achse $\frac{1}{2} \cdot r_{1}$ gewählt. Die Indizes für die Atomlagen und die berechneten Nullpunktsabstände für die Atome auf der 0. bis 4. horizontalen Atomgerade sind in Tab. 2 zusammengestellt. Die Nullpunktsabstände, welche auch experimentell erhalten wurden, sind fett gedruckt. Bei einem Vergleich der Tabellen 1 und 2 stellt man fest, daß die ersten beiden Atomabstände $r_{1}=2,94 \AA$ und $r_{1} \sqrt{3}=5,09 \AA$ der Kugelmodell-Struktur wegen Überlagerung der zugehörigen Maxima mit denen der Atomabstände $r_{1}^{\prime}=2,72 \AA$ und $2 r_{1}^{\prime}=5,44 \AA$ der FlächengitterStruktur vom Experiment nicht gesondert geliefert werden. Beim $r_{1} \sqrt{7}$-Abstand ist kaum eine $\mathrm{Ab}$ weichung vom berechneten Werte festzustellen, bei den weiteren Abständen macht sich wie bei festem amorphem As, Ge, Se u. a. m. eine zunehmende Verkürzung der gemessenen Atomabstände gegenüber den berechneten bemerkbar (vgl. Richter und BREITLING ${ }^{11}$ ). Nach den Tabellen 1 und 2 stimmen die experimentell erhaltenen Atomabstände mit den berechneten Nullpunktsabständen der Atome auf der

\begin{tabular}{|c|c|c|c|c|}
\hline 0. Atomgerade & 1. Atomgerade & 2. Atomgerade & 3. Atomgerade & 4. Atomgerade \\
\hline $20 \triangleq r_{1} \sqrt{3}=5,09$ & $11 \triangleq r_{1} \quad=2,94$ & $02 \triangleq r_{1}$ & $13 \triangleq r_{1} \sqrt{3}=5,09$ & $04 \stackrel{2}{2 r_{1}}$ \\
\hline $40 \triangleq 2 r_{1} \sqrt{3}=10,18$ & $31 \triangleq r_{1} \sqrt{7}=7,78$ & $22 \triangleq 2 r_{1} \quad=5,88$ & $33 \triangleq 3 r_{1} \quad=8,82$ & $24 \triangleq r_{1} \quad \sqrt{7}$ \\
\hline $60 \triangleq 3 r_{1} \sqrt{3}=15,27$ & $51 \triangleq r_{1} \sqrt{19}=12,81$ & $42 \triangleq r_{1} \sqrt{13}=10,60$ & $53 \stackrel{=}{=} r_{1} \sqrt{21}=13,49$ & 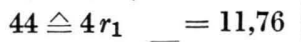 \\
\hline $80 \triangleq 4 r_{1} \sqrt{3}=20,36$ & $71 \triangleq r_{1} \sqrt{37}=17,88$ & $62 \triangleq 2 r_{1} \sqrt{7}=15,56$ & $73 \triangleq r_{1} \sqrt{39}=18,36$ & $64 \triangleq r_{1} \sqrt{31}=16,37$ \\
\hline
\end{tabular}

Tab. 2. Indizes und berechnete Nullpunktsabstände der Atome auf der 0. bis 4. Atomgerade der Abb. 3 für $r_{1}=2,94 \AA$.

11 H. Richter u. G. Breitling, Z. Naturforschg. 6 a,721 [1951]; 13 a, 988 [1958]. 
0 . Atomgerade mit $y=0$ ( $x$-Achse $\triangleq r_{1} \sqrt{3}$-Richtung $\triangleq$ [100]-Richtung) und auf der 1. Atomgerade mit $y=1$ überein. Schon die Nullpunktsabstände der Atome auf der 2. Atomgerade mit $y=2$ werden vom Experiment nicht geliefert, insbesondere auch nicht der doppelte Atomabstand $0 \rightarrow 22=2 r_{1}=5,88 \AA$ der Kugelmodell-Struktur. Ebenso zeichnen sich die Nullpunktsabstände $r_{\nu}=\nu \cdot r_{1}$ der Atome in der $r_{1}$-Richtung ( $y$-Achse $\triangleq[010]$-Richtung), auf der sich die Atome unmittelbar berühren, in Abb. 2 nicht ab; das gleiche gilt für die Atome in der [110]-Richtung. Beide Richtungen besitzen nämlich dieselben Atomlagen und damit die gleichen Atomabstände, sie sind gleichwertig.

In Abb. 2 treten lediglich die Nullpunktsabstände der Atome der 0. und der 1. Atomgerade auf. Damit bildet sich die Zickzackkette mit $\varphi=120^{\circ}$ der Abb. 3 in der Atomverteilungskurve von geschmolzenem Ag ab. Mit der $r_{1} \sqrt{3}$-Richtung als Vorzugsrichtung ${ }^{*}$ entziehen sich die zugehörigen Seitenatome auf den höher indizierten Atomgeraden wegen der zunehmenden Abstandsschwankungen der Beobachtung. So wird hier, wie sooft bei den nichtkristallinen Stoffen, das Bild einer Atomkette vorgetäuscht, während in Wirklichkeit eine gestörte Flächengitter-Struktur vorliegt, und zwar bei $\mathrm{Ag}$ mit der $r_{1} \sqrt{3 \text {-Richtung als }}$ Faltungsrichtung. Die Flächengitter müssen offenbar parallel zu dieser Richtung geringfügig gefaltet bzw. gewellt sein (vgl. Richter und Breitling ${ }^{11,3}$ ). Bei diesem Faltungsvorgang bleiben nämlich die Atomabstände innerhalb der Zickzackkette erhalten. Die Zickzackkette ist demnach keine Baueinheit der Metallschmelze, sie stellt vielmehr einen Teil eines größeren Bereiches mit begrenzter Ordnung dar.

Im Gegensatz zur Ag-Schmelze treten in den Schmelzen von Sn nach den Abb. 1 a und b sowie von $\mathrm{Bi}$ und Ga die Atomabstände $\boldsymbol{r}_{\boldsymbol{v}}=\boldsymbol{v} \cdot \boldsymbol{r}_{1}$ der dichtesten Kugelpackung auf (vgl. Richter und Breitling ${ }^{12}$ ). Diese Atomabstände lassen sich ebenfalls an Hand

* Teile dieser Arbeit wurden auf der Gordon Research Conference on thin films 1966 in Tilton/N. H., USA, vorgetragen. Bei dieser Gelegenheit machte uns Frau Prof. D. KuHLMANN-WILsDorf, University of Virginia, USA, darauf aufmerksam, daß bei Ag die Vorzugsrichtung $r_{1} \sqrt{3}$ mit der Gitterrichtung [112] identisch ist und durch Versetzungen erklärt werden kann vgl. Breitling, KUHLMANN-WilsDorf u. Richter, J. Chem. Phys. 1967).

12 H. Richter u. G. Breitling, Z. Naturforschg. 20 a, 1061 [1965]. der Abb. 3 erklären, und zwar mit der [010]- bzw. [110]-Richtung als Vorzugsrichtung. Dabei ist es notwendig, daß die seitlich dazu gelegenen Atome starke Abstandsschwankungen erfahren. Wahrscheinlicher ist vielleicht die folgende Deutung. Bei geschmolzenem Sn, Bi und $\mathrm{Ga}$ ist in den Bereichen der dichtesten Kugelpackung eine räumliche Anordnung mit deutlichen Lagestreuungen der Atome vorhanden ähnlich wie bei der Flächengitter-Struktur mit den bevorzugten Atomabständen $r_{1}{ }^{\prime}, 2 r_{1}{ }^{\prime}, 3 r_{1}{ }^{\prime}$ usf. Dabei zeigen auch hier die Atome, welche bei lückenloser Anordnung und geringer seitlicher Streuung praktisch auf Geraden liegen, die geringsten Abstandsschwankungen. Diese Atome bzw. deren Atomabstände ${ }^{13} r_{1}, 2 r_{1}, 3 r_{1}$ usf. bilden sich daher im Streubild bzw. in der Atomverteilungskurve diskret ab. Wegen der starken Abstandsschwankungen der Seitenatome haben allein der kürzeste Atomabstand $r_{1}$ und dessen Vielfache eine Chance, sich abzuzeichnen. Danach besitzt die dichteste Kugelpackung in den Schmelzen von Sn, Bi und Ga einen geringeren Ordnungsgrad als in den Schmelzen von $\mathrm{Au}, \mathrm{Ag}, \mathrm{Pb}$ und $\mathrm{Tl}$.

Nach diesen Ergebnissen ist in der Ag-Schmelze selbst im Falle der dichtesten Kugelpackung keine statistisch isotrope Verteilung der Atome vorhanden, man hat stattdessen eine Flächengitter-Siruktur mit hexagonaler Gitterzelle anzunehmen. Damit ist die Ag-Schmelze ein Haufwerk von Flächengittern mit hexagonaler und mit quadratischer Gitterzelle; Gleiches gilt für die Schmelzen von $\mathrm{Au}, \mathrm{Pb}$ und $\mathrm{Tl}$.

Nach unveröffentlichten Neutronenbeugungsversuchen von Richter und Oenme ${ }^{15}$ findet man selbst bei den Alkalimetallschmelzen ähnliche Verhältnisse vor. Im vorderen Teile der $4 \pi r\left[\varrho(r)-\varrho_{0}\right]$-Kurven von geschmolzenem $\mathrm{Na}$ und $\mathrm{Cs}$ sind die beiden Gitterabstände $\left[r_{1}\right]_{\mathrm{G}}=\frac{1}{2} a_{\mathrm{W}} \sqrt{3}$ und $\left[r_{5}\right]_{\mathrm{G}}=a_{\mathrm{W}} \sqrt{3}$ sowie die beiden Atomabstände $r_{1}$ und $r_{1} \sqrt{3}$ der dichtesten Kugelpackung wirksam. Dabei wird in der Nähe des

13 Bei festem amorphem und bei geschmolzenem $\mathrm{Bi}$ treten vier Maxima in der $4 \pi r^{2} \varrho(r)$-Kurve äquidistant auf (vgl. Leonhardt, Richter und Rossteutscher ${ }^{14}$ ), und zwar folgen sie mit $r_{1}=3,32 \AA$ als kürzestem Atomabstand der dichtesten Kugelpackung aufeinander; hier ist also $r_{\nu}=\nu \cdot r_{1}$; Gleiches trifft für das feste amorphe und für das unterkühlte Ga zu. Wie bei geschmolzenem $\mathrm{Sn}$ in den Abb. 1 a und $\mathrm{b}$ tritt auch bei festem amorphem und bei geschmolzenem Bi und $\mathrm{Ga}$ der $r_{1} \sqrt{3-A b s t a n d}$ nicht auf.

14 R. Leonhardt, H. Richter u. W. Rossteutscher, Z. Phys. 165, 121 [1961].

15 H. Richter u. H. Oehme, Naturwiss. 1967; Advances in Physics 1967. 
Schmelzpunktes das I. Maximum vom kürzesten Gitterabstande $\left[r_{1}\right]_{\mathrm{G}}=\frac{1}{2} a_{\mathrm{w}} \sqrt{3}$ fixiert; hierbei ist, besonders bei $\mathrm{Na}$, der Atomabstand $r_{1}$ mitbestimmend. Das II. Maximum wird entsprechend von den Atomabständen $a_{\mathrm{w}} \sqrt{3}$ und $r_{1} \sqrt{3}$ festgelegt. Der Einflu $\beta$ des $r_{1} \sqrt{3}$-Abstandes wächst mit zunehmender Ordnungszahl, so daß bei Cs das II. Maximum praktisch vom Atomabstand $r_{1} \sqrt{3}$ bestimmt wird. Das 3. Maximum und die weiteren Maxima werden wie bei geschmolzenem $\mathrm{Au}, \mathrm{Ag}, \mathrm{Pb}$ und $\mathrm{Tl}$ vom $r_{1} \sqrt{7}$. Abstand - hier ist wieder eine volle Übereinstimmung zwischen Experiment und Berechnung vorhanden - und von den Atomabständen $2 r_{1} \sqrt{3}$, $r_{1} \sqrt{19} \ldots$ festgelegt. Auch in den $4 \pi r\left[\varrho(r)-\varrho_{0}\right]$ Kurven von $\mathrm{Na}$ und $\mathrm{Cs}$ kommt der doppelte Atomabstand $2 r_{1}$ der dichtesten Kugelpackung nicht vor.

In den $4 \pi r\left[\varrho(r)-\varrho_{0}\right]$-Kurven von $\mathrm{Au}, \mathrm{Ag}, \mathrm{Pb}$ und $\mathrm{Tl}$ sowie von $\mathrm{Na}$ und $\mathrm{Cs}$ zeichnet sich $\operatorname{der} r_{1} \sqrt{7}$ Abstand im 3. Maximum sehr deutlich ab. Offensichtlich ist hier der Einfluß der Flächengitter-Struktur, bei Na und Cs der Einfluß der Raumgitterstruktur, nach dem II. Maximum abgeklungen, andererseits macht sich die Abstandsverkürzung, die durch die Lagestreuung der Atome verursacht ist, noch nicht bemerkbar. Bei Au, Ag, $\mathrm{Pb}$ und $\mathrm{Tl}$ sowie bei den $\mathrm{Al}$ kalimetallen erhält man $4 \pi r\left[o(r)-\varrho_{0}\right]$-Kurven mit ähnlichem Aussehen; sie sind entsprechend dem Atomabstand verkürzt oder gedehnt. Die Alkalimetallschmelzen stehen damit dem geschmolzenen $\mathrm{Au}, \mathrm{Ag}$, $\mathrm{Pb}$ und $\mathrm{Tl}$ sehr nahe, weniger dagegen dem geschmolzenen Sn, Ga und Bi.

\section{Unterschiede im Aufbau der Ag- und der Sn-Schmelze}

Die Diskussion der Intensitätskurve, insbesondere die neuen Auswertungsverfahren, haben gezeigt, wie die Intensitätskurve zustandekommt, und welche Atomabstände am Streuvorgange beteiligt sind; sie haben schließlich zu der Vorstellung geführt, daß zwei Strukturen, und zwar die eine mit metallischer und die andere mit homöopolarer Bindung, in kleinsten Bereichen der Metallschmelze nebeneinander vorkommen. Die Kenntnis dieser Doppelstruktur hat die Diskussion der $4 \pi r\left[\varrho(r)-\varrho_{0}\right]$-Kurven in den Abb. 1 a und b sowie in Abb. 2 wesentlich erleichtert.

Bei geschmolzenem Ag wird nach Abb. 2 das I. Maximum von den kürzesten Atomabständen $r_{1}$ und $r_{1}{ }^{\prime}$ der Kugelmodell- und der Flächengitter-Struktur bestimmt, das II. Maximum wird entsprechend vom $r_{1} \sqrt{3}$-Abstand der dichtesten Kugelpackung und vom doppelten Atomabstande $2 r_{1}{ }^{\prime}$ der FlächengitterStruktur festgelegt. Das 3. Maximum und die weiteren Maxima werden dagegen allein von den Atomabständen der Zickzackkette der dichtesten Kugelpackung bestimmt. Nach Abb. 2 ist nämlich der Einfluß der Flächengitter-Struktur mit quadratischer Gitterzelle bereits mit dem II. Maximum abgeklungen. Bei geschmolzenem Sn von $250^{\circ}$ und $750{ }^{\circ} \mathrm{C}$ liegen die Verhältnisse grundsätzlich anders. Hier wird bei $250{ }^{\circ} \mathrm{C}$ nach Abb. 1 a das I. Maximum praktisch vom einfachen Atomabstande $r_{1}$ und das II. Maximum vom doppelten Atomabstande $2 r_{1}$ der dichtesten Kugelpackung fixiert, bei $750{ }^{\circ} \mathrm{C}$ sind nach Abb. $1 \mathrm{~b}$ an der Festlegung des I. sowie des II. Maximums die einfachen bzw. die doppelten Atomabstände $r_{1}$ und $r_{1}{ }^{\prime}$ bzw. $2 r_{1}$ und $2 r_{1}{ }^{\prime}$ der Kugelmodell- und der Flächengitter-Struktur beteiligt. Das 3. und das 4. Maximum werden in beiden Fällen allein von der Flächengitter-Struktur mit ihrer starken homöopolaren Bindung bestimmt. Diese Flächengitterbereiche mit Debye-Streuung sind bei Sn weit ausgedehnter als die Bereiche der Kugelmodell-Struktur, zudem kommen hier selbst bei hohen Temperaturen noch Flächengitter mit LAUE-Streuung vor, also Teilbereiche des Sn-Gitters. Bei Ag dagegen sind nach Abb. 2 die Bereiche der Kugelmodell-Struktur in Form von Flächengittern mit hexagonaler Gitterzelle ausgedehnter als die der Flächengitter-Struktur mit quadratischer Gitterzelle. All' diese Verhältnisse sind für die Kristallbildung aus der Metallschmelze wichtig und mitbestimmend (vgl. Richter und BreitLING ${ }^{9,16}$ ).

Als neues Ergebnis zeigen die Abb. 1 a und $b$, daß in geschmolzenem Sn die Atomabstände $r_{v}{ }^{\prime}$ der Flächengitter-Struktur mit Deвуе-Streuung Vielfache des Grundabstandes $r_{1}{ }^{\prime} \approx\left[r_{1}\right]_{\mathrm{G}}$ sind, $\mathrm{d}$. $\mathrm{h}$. es ist $r_{v}{ }^{\prime}=\boldsymbol{v} \cdot r_{1}{ }^{\prime}$. Damit ist in diesen Bereichen der Schmelze das Sn-Gitter nicht mehr vorhanden. Die Flächengitter bestehen bei geschmolzenem $\mathrm{Sn}$ und $\mathrm{Bi}$, sicherlich auch bei den anderen Schmelzen mit homöopolarer Bindung im Gitter wie $\mathrm{Hg}, \mathrm{Ga}, \mathrm{Ge}, \mathrm{Sb}$ u. a.m., aus einer quadratischen Gitterzelle mit $r_{1}^{\prime} \approx\left[\mathrm{r}_{1}\right]_{\mathrm{G}}$ als Kantenlänge (bezüglich In vgl. Knosp ${ }^{17}$ ). Die gleichen Verhältnisse liegen bei geschmolzenem Au, Ag, Pb, Tl, In u. a. m. vor, wo die Flächengitter ebenfalls eine quadratische Gitterzelle

16 H. Richter u. G. Breitling, Z. Naturforschg. 18 a, 23 [1963]. 
besitzen, allerdings ist hier $r_{1}{ }^{\prime}<\left[r_{1}\right]_{\mathrm{G}}=r_{12}$. So konnten Richter und Breitling ${ }^{18}$ bei $\mathrm{Au}$ und $\mathrm{Ag}$ zeigen, da $\beta$ in den Atomverteilungskurven der $r_{1}{ }^{\prime} \sqrt{2 \text { - }}$ Abstand mit $r_{1}{ }^{\prime}=2,72 \AA$ vorkommt

$$
\left(r_{1}{ }^{\prime} \sqrt{2}=2,72 \sqrt{2} \AA=3,85 \AA\right) .
$$

Hier hat man bei der Flächengitter-Struktur ebenfalls mit dem Auftreten der Atomabstände $r_{v}{ }^{\prime}=v \cdot r_{1}{ }^{\prime}$ $\mathrm{zu}$ rechnen. Neuerdings fanden Leonhardt und Richter ${ }^{19}$ bei abschnittsweiser Fourier-Analyse der $i(s)_{\text {exp. }}$-Kurve von geschmolzenem $\mathrm{Tl}$ die Atomabstände $r_{1}{ }^{\prime}, 2 r_{1}{ }^{\prime}$ und $3 r_{1}{ }^{\prime}$, also Vielfache des kürzesten Atomabstandes $r_{1}{ }^{\prime}=3,25 \AA$ der FlächengitterStruktur. Gleiches stellte KNosP ${ }^{17}$ bei geschmolzenem In fest, wo die Flächengitter-Struktur mit $r_{1}{ }^{\prime}=3,02 \AA$ besonders stark vertreten ist. Bei $\mathrm{Tl}$ und $\mathrm{In}$ ist somit wiederum $r_{v}{ }^{\prime}=v \cdot r_{1}{ }^{\prime}$.

Weiter ist nach Abb. 2 neu, daß bei der dichtesten Kugelpackung nur die Atomabstände ${ }^{20} r_{1}, r_{1} \sqrt{3 \text {, }}$ $r_{1} \sqrt{7}, r_{1} \sqrt{19}, r_{1} \sqrt{37} \ldots$ und Vielfache von $r_{1} \sqrt{3}$ auftreten (vgl. Tab. 2 und Abb. 3). Danach kommt die dichteste Kugelpackung in geschmolzenem $\mathrm{Au}, \mathrm{Ag}$, $\mathrm{Pb}$ und $\mathrm{Tl}$ lediglich in Form von Flächengittern mit

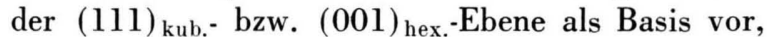
und zwar bei bestimmter Lagestreuung der Atome. So bauen sich die Schmelzen von $\mathrm{Au}, \mathrm{Ag}, \mathrm{Pb}$ und $\mathrm{Tl}$ aus Flächengittern mit hexagonaler und mit quadratischer Gitterzelle auf; sie sind Haufwerke zweier Arten von Flächengittern, die in einem von der Temperatur abhängigen Verhältnis nebeneinander vorkommen. In den Schmelzen von $\mathrm{Sn}, \mathrm{Ga}$ und $\mathrm{Bi}$ treten hinsichtlich der dichtesten Kugelpackung lediglich die Atomabstände $r_{v}=\boldsymbol{v} \cdot r_{1}$ auf. Die Flächengitter-Struktur dagegen besteht hier wie bei geschmolzenem $\mathrm{Au}, \mathrm{Ag}, \mathrm{Pb}$ und $\mathrm{Tl}$ aus einer quadratischen Gitterzelle.

\section{Festes amorphes Selen}

Als Beispiel für das Auftreten einer realen Zickzackkette sei in Abb. 4 die Atomverteilungskurve von festem amorphem Se, diesmal in Gestalt der

17 H. Knosp, Diplom-Arbeit, Technische Hochschule Stuttgart 1964. In gehört bezüglich der Flächengitter-Struktur mit $r_{1}^{\prime}<\left[r_{1}\right]_{G}$ zur Gruppe I, wegen des Fehlens des $r_{1} \sqrt{3}-\mathrm{Ab}$ standes der dichtesten Kugelpackung jedoch zur Gruppe II; es steht somit am Übergang beider Gruppen (vgl. den Tagungsbeitrag von Richter und Breithing zur Conference on properties of liquid metals, Brookhaven National Laboratory in Upton, USA, publiziert in Advances in Physics 1967). Hg ist eindeutig der Gruppe II zuzuordnen.

18 H. Richter u. G. Breitling, Z. Naturforschg. 16 a, 187[1961].

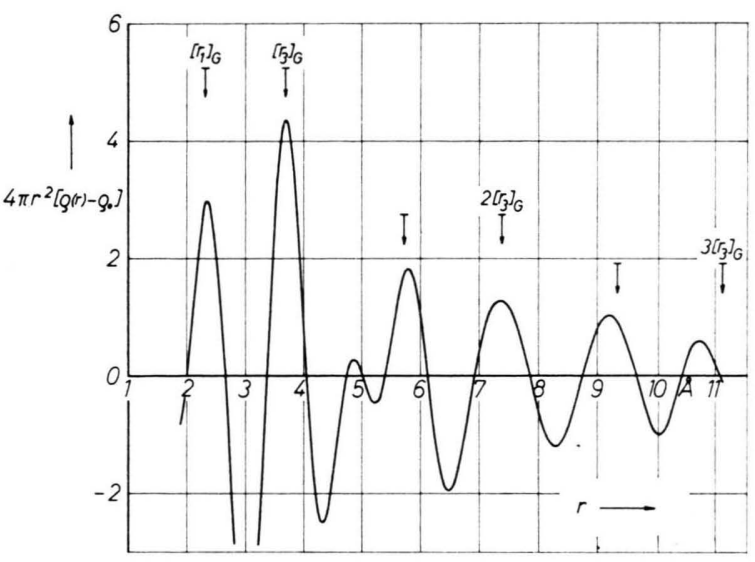

Abb. 4. 4 $\pi r^{2}\left[\varrho(r)-\varrho_{0}\right]$-Kurve von festem amorphem Selen. Pfeile $\bar{\downarrow}=$ berechnete Atomabstände innerhalb der ebenen Se-Kette mit $\left[r_{1}\right]_{\mathrm{G}}=2,32 \AA$ und $\left[r_{3}\right]_{\mathrm{G}}=3,69 \AA \quad\left(\varphi=105^{\circ}\right)$ als Abmessungen des Elementardreiecks.

$4 \pi r^{2}\left[\varrho(r)-\varrho_{0}\right]-$ Kurve, betrachtet (vgl. Breituing und Richter ${ }^{3}$ ). Die ersten beiden Maxima bei $r_{1}=2,34 \AA$ und $r_{3}=3,69 \AA$ stimmen mit den Gitterabständen $\left[r_{1}\right]_{\mathrm{G}}=2,32 \AA$ und $\left[r_{3}\right]_{\mathrm{G}}=3,69 \AA$ überein. Weitere Maxima treten bei $r=5,76 ; 7,36 ; 9,19$; $10,70 \AA$ usf. auf. Nimmt man in festem amorphem Se eine ebene Kette an, die sich aus den Gitterabständen $\left[r_{1}\right]_{\mathrm{G}}=2,32 \AA(\bar{\downarrow})$ und $\left[r_{3}\right]_{\mathrm{G}}=3,69 \AA(\downarrow)$ [Bindungswinkel $\varphi=105^{\circ}$ ] aufbaut, so folgen als weitere Atomabstände innerhalb der Einzelkette ${ }^{21}$ $r=5,71 ; 7,38 ; 9,33 ; 11,07 \AA$ usf. Diese Abstandswerte stehen ebenfalls mit den experimentellen Maximalagen in bestem Einklang. Bei großen Atomabständen macht sich wegen der Lagestreuung der Atome auch hier wie bei den Metallschmelzen eine zunehmende Abstandsverkürzung gegenüber den berechneten Werten bemerkbar. Aus dem Auftreten des doppelten Atomabstandes $2\left[r_{3}\right]_{\mathrm{G}}=2 \cdot 3,69 \AA$ $=7,38 \AA$ folgt die Existenz der ebenen Se-Kette. Die überragende Höhe des II. Maximums gegenüber dem 1. Maximum ist durch den kürzesten (ersten) Kettenabstand $r_{2}=r_{\mathrm{K} 1}=3,85 \AA$ bedingt (Kettenabstand $=$ Abstand der Atome von Kette zu Kette).

19 R. Leonhardt u. H. Richter, Z. Naturforschg. 21 a, 179 [1966].

20 Nach S. 1713/15 kommen die Atomabstände $r_{v}=v \cdot r_{1}$ mit $v \geq 2$ sowie die Atomabstände $r_{1} \sqrt{13}, r_{1} \sqrt{21}, r_{1} \sqrt{31}$, usf. nicht vor. Damit fehlen alle Nullpunktsabstände der Atome auf den Atomgeraden mit $y \geqq 2$. In der Atomverteilungskurve zeichnet sich daher lediglich die Zickzackkette ab, die von den Atomen der 0 . und der 1. Atomgerade $(y=0$ und $y=1)$ der Abb. 3 gebildet wird.

21 Die Atomabstände in der Schraubenkette des Se-Gitters sind $[r]_{\mathrm{G}}=2,32 ; 3,69 ; 4,95 ; 6,80 ; 8,41 \AA$ usf. 
Die Se-Ketten sind in diesem Abstande zu Schichten (Flächengitter) aneinandergelagert. Der kürzeste Kettenabstand im Se-Gitter beträgt $\left[r_{2}\right]_{\mathrm{G}}=3,46 \AA$. Mit $r_{\mathrm{K} 1}=3,85 \AA$ errechnet man als zweiten Kettenabstand $r_{\mathrm{K} 2}=4,78 \AA$; experimentell findet man im Mittel $r_{\mathrm{K} 2}=4,80 \AA$.

Nach der Fourier-Analyse der Intensitätskurve liegt in festem amorphem Se eine einzige Struktur vor; sie baut sich aus Flächengittern auf, die ihrerseits aus ebenen Atomketten bestehen. Die ebene SeKette erklärt die geringe Neigung des aufgedampften amorphen und des glasig erstarrten Se zur Gitterbildung.

Das feste amorphe Se ist gitterähnlich, das feste amorphe $\mathrm{Ga}$ und $\mathrm{Bi}$ sind dagegen flüssigkeitsähnlich, letztere bauen sich wie die Metallschmelze aus zwei Strukturen, der Kugelmodell- und der FlächengitterStruktur, auf.

Selbst bei festem amorphem $\mathrm{Si}, \mathrm{Ge}, \mathrm{As}$ und $\mathrm{Sb}$ zeichnet sich nach Richter und Breituing ${ }^{11}$ lediglich die Zickzackkette in der Atomverteilungskurve ab. Hier entziehen sich die Seitenatome infolge ihrer größeren Abstandsschwankungen der Beobachtung, so daß wieder das Bild der Atomkette erhalten wird. Eine Abweichung der gemessenen Abstandswerte von den berechneten setzt bei $\mathrm{Si}, \mathrm{Ge}, \mathrm{As}$ und $\mathrm{Sb}$ im Gegensatz zu den Metallschmelzen und zu festem amorphem Se (vgl. die Abb. 2 und 4) meistens schon beim 3. Maximum der $4 \pi r\left[\varrho(r)-\varrho_{0}\right]$-Kurve ein, was als Folge der Schwankungen des Bindungswinkels $\varphi$ zu deuten ist.

Nach der $4 \pi r\left[\varrho(r)-\varrho_{0}\right]$-Darstellung der Atomdichte geschmolzener (einatomiger) Metalle tragen bestenfalls 8 Atomabstände zum Streubild bei, davon legen höchstens 4 Atomabstände bzw. Interferenzfunktionen mit ausgeprägten Oszillationen und mit unterschiedlicher Dämpfung das differenzierte Streubild, den charakteristischen Verlauf der Intensitäts- bzw. der $i(s)$ exp.-Kurve, fest. Die Folge der Atomabstände von einem geordneten Bereich zum nächsten Übergangsgebiet mit seiner ungeordneten Atomverteilung oder von einem geordneten Bereich zum nächsten geordneten Bereich ist kontinuierlich (kontinuierliches Abstandsspektrum). Dieses Abstandsspektrum liefert lediglich zur Atomstreuung und damit zur gleichmäßigen bzw. zur regellosen Atomverteilung einen Beitrag, nicht aber zur diskreten Atomverteilung (vgl. Herre und Richter ${ }^{2}$ ).

Herrn Dipl.-Phys. R. Leonhardt danken wir herzlich für die vielen Fourier-Analysen, die er im Rahmen dieser Arbeiten durchgeführt hat, auch sei der Deutschen Forschungsgemeinschaft für apparative Unterstützung dieser Untersuchungen gedankt.

\section{BERICHTIGUNG}

$\mathrm{Zu}:$ H. J. GläsER and D. GeIST, Electron Paramagnetic Resonance of $\mathrm{Eu}^{2+}$ in $\mathrm{CdF}_{2}$, Vol. 20 a, 842 [1965].

The values in the first line of Tab. 1 are to be corrected as follows $g=1,9918 \pm 0,0005, A\left(\mathrm{Eu}^{151}\right)=(33,9 \pm 0,15) \cdot 10^{-4} \mathrm{~cm}^{-1}, A\left(\mathrm{Eu}^{153}\right)=(15,05 \pm 0,15) \cdot 10^{-4} \mathrm{~cm}^{-1}$, $b_{4}=(52,37 \pm 0,1) \cdot 10^{-4} \mathrm{~cm}^{-1},-b_{6}=(0,24 \pm 0,05) \cdot 10^{-4} \mathrm{~cm}^{-1}$.

(Signs and the corresponding values for $77^{\circ} \mathrm{K}$ and $1,5^{\circ} \mathrm{K}$ will be published soon.) 